\title{
Coupling-Matrix Approach to the Chern Number Calculation in Disordered Systems
}

\author{
Y. F. Zhang ${ }^{1}$, Y. Y. Yang ${ }^{1}$, Yan Ju ${ }^{1}$, L. Sheng*1, R. Shen ${ }^{1}$, D. \\ N. Sheng ${ }^{2}$, D. Y. Xing ${ }^{\dagger 1}$ \\ ${ }^{1}$ National Laboratory of Solid State Microstructures and Department of Physics, \\ Nanjing University, Nanjing 210093, China \\ ${ }^{2}$ Department of Physics and Astronomy, California State University, Northridge, \\ California 91330, USA \\ E-mail: * shengli@nju.edu.cn, $\dagger$ dyxing@nju.edu.cn
}

\begin{abstract}
The Chern number is often used to distinguish between different topological phases of matter in two-dimensional electron systems. A fast and efficient coupling-matrix method is designed to calculate the Chern number in finite crystalline and disordered systems. To show its effectiveness, we apply the approach to the Haldane model and the lattice Hofstadter model, the quantized Chern numbers being correctly obtained. The disorder-induced topological phase transition is well reproduced, when the disorder strength is increased beyond the critical value. We expect the method to be widely applicable to the study of topological quantum numbers.
\end{abstract}

PACS numbers: 73.43.Nq, 71.23.An, 72.80.Vp 


\section{Introduction}

In the past thirty years, the condensed matter physics community has been fascinated by topological phases of matter, for instance, the integer quantum Hall effect, [1] the fractional quantum Hall effect, [2] the quantum anomalous Hall effect, [3] the quantum spin Hall effect, [4, 5] and the three-dimensional topological insulators. [6, 7] These topological states of quantum matter are usually distinguished by use of some global topological quantum numbers [8] rather than certain local order parameters. The topological aspect of the integer quantum Hall effect with periodic potentials was first discussed by Thouless, Kohmoto, Nightingale, and Nijs (TKNN). [9] In their famous work, a topological expression for the Hall conductivity was given by the Chern number over the magnetic Brillouin zone. Their result was then generalized to the fractional quantum Hall effect. [10] For the quantum spin Hall systems, with the extension of the idea, the well-defined spin Chern number can be used to characterize trivial and non-trivial bulk band topology. [11, 12]

While simplification exists for pure systems [13], calculation of the Chern number in the presence of disorder is usually based upon the integral of partial derivatives of electron wave functions over the boundary phases. [10, 14, 15] Numerical implementation involves hundreds of times of exact diagonalization for a given disorder configuration [14, 15, 16, 17, which is very time-consuming even for noninteracting electron systems. Recently, several different approaches for the Chern number computation in real space have been suggested in the literature. Kitaev [18] proposed a real space Chern number formula for a lattice model in terms of traces of the coordinate operator and projection operator. A recent work similar to Kitaev's one was numerically realized by Bianco and Resta [19], and they generalized the result to the systems with open boundary conditions. In the presence of disorder, however, the idea of supercells, 20] namely, a periodic duplication of the actual system is needed, which greatly increases the computation time. Based upon the noncommutative Chern number theory, Prodan et al. [21] proposed an efficient method to calculate the Chern number of disordered systems, in which the procedure of the exact diagonalization is greatly simplified. However, it involves somewhat complicated multiple commutators between the coordinate operators and projection operators. In some cases, the Chern number can be extracted indirectly from the transport coefficients, which can be relatively easily calculated from the Kubo formula [16]. In some other cases, direct calculation of the topological number of bulk wavefunctions is often needed and sometimes irreplaceable. Therefore, development of efficient numerical approaches to direct calculation of the Chern number is highly desirable.

In this work, we provide an alternative way to calculate the Chern number, in which only one time exact diagonalization for the actual system is needed without loss of accuracy. A transparent coupling-matrix formulation will be given, from which the Chern number can be very efficiently computed, compared with the existing approaches. To show its effectiveness, this approach is applied to both the Haldane model and the 
Hofstadter model. The calculated Chern number is found well quantized provided the Fermi level lies within the energy gap, even when the sample size is not very large. The topological phase transition from the quantum Hall insulator to an ordinary insulator can be determined based upon the calculated Chern number, and the obtained critical disorder strength is in good agreement with the result previously obtained from the Hall conductivity calculation.

In the next section, we present the new approach of calculating the Chern number. In Sec. III, for the Haldane model, it is shown that the present approach works well for both crystalline and disordered systems. In Sec. IV, we apply the approach to the lattice Hofstadter model and the calculated results are in accordance with already existing results. The final section is a summary.

\section{Method}

We now consider a two dimensional (2D) lattice with $N=L_{x} \times L_{y}$ unit cells. We use $\mathbf{r}=(x, y)$ with $x$ and $y$ as integers to index the position of a unit cell. We can define twisted boundary conditions for a single-particle wavefunction of the system $\varphi_{\theta}\left(x+L_{x}, y\right)=e^{i \theta_{x}} \varphi_{\theta}(x, y)$ and $\varphi_{\theta}\left(x, y+L_{y}\right)=e^{i \theta_{y}} \varphi_{\theta}(x, y)$ with $\theta=\left(\theta_{x}, \theta_{y}\right)$ and $0 \leq \theta_{x}, \theta_{y} \leq 2 \pi$. The wavefunction is in general a row vector in the space of inner degrees of freedom, such as spin and sublattice. The system is assumed to have $M$ electrons, and in the ground state the wavefunctions of the $M$ occupied single-particle electron states are denoted by $\varphi_{\theta}^{m}(\mathbf{r})$ with $m=0,1, \cdots M-1$. The many-body wavefunction of the ground state $\Psi_{\theta}\left(\left\{\mathbf{r}_{i}\right\}\right)$ is the Slater determinant of the single-particle wavefunctions $\varphi_{\theta}^{m}\left(\mathbf{r}_{i}\right)$, where $\mathbf{r}_{i}$ with $i=0,1, \cdots M-1$ is the coordinate of the $i$-th electron. The Chern number of the gound state is given by [10, 15, 14]

$$
C=\frac{1}{2 \pi i} \int_{T_{\theta}} d \theta\left\langle\nabla_{\theta} \Psi_{\theta}|\times| \nabla_{\theta} \Psi_{\theta}\right\rangle
$$

with $\theta=\left(\theta_{x}, \theta_{y}\right)$ and $T_{\theta}$ denoting the $\left(\theta_{x}, \theta_{y}\right)$ space, which is essentially a torus.

We transform the single-particle eigenstate from real space to momentum space through a Fourier transform (FT)

$$
\varphi_{\theta}^{m}\left(\mathbf{r}_{i}\right)=\frac{1}{\sqrt{N}} \sum_{\mathbf{k}_{i}} F^{m}\left(\mathbf{k}_{i}\right) e^{i \mathbf{k}_{i} \cdot \mathbf{r}_{i}} .
$$

The twisted boundary conditions require that the momenta take only the discrete values $\mathbf{k}_{i}=\mathbf{k}_{i}^{(0)}+\mathbf{q}$, where $\mathbf{k}_{i}^{(0)}=\left(\frac{2 n \pi}{L_{x}}, \frac{2 l \pi}{L_{y}}\right)$ with $0 \leq n<L_{x}$ and $0 \leq l<L_{y}$, and $\mathbf{q}=\left(\frac{\theta_{x}}{L_{x}}, \frac{\theta_{y}}{L_{y}}\right)$. We note that the set of $\left\{\mathbf{k}_{i}^{(0)}\right\}$ are actually the discrete momenta for periodic boundary conditions. We will denote $F^{m}\left(\mathbf{k}_{i}\right) \equiv F^{m}\left(\mathbf{k}_{i}^{(0)}+\mathbf{q}\right)$ as $F_{\mathbf{q}}^{m}\left(\mathbf{k}_{i}^{(0)}\right)$. It is easy to find that the many-body wavefunction of the ground state in the momentum space $\Phi_{\mathbf{q}}\left(\left\{\mathbf{k}_{i}^{(0)}\right\}\right)$ is the Slater determinant of $F_{\mathbf{q}}^{m}\left(\mathbf{k}_{i}^{(0)}\right)$. By means of the substitution $\frac{\partial}{\partial \theta_{x}} \rightarrow \frac{1}{L_{x}} \frac{\partial}{\partial q_{x}}$ and $\frac{\partial}{\partial \theta_{y}} \rightarrow \frac{1}{L_{y}} \frac{\partial}{\partial q_{y}}$, the Chern number is derived to be

$$
C=\frac{1}{2 \pi i} \int_{R_{\mathbf{q}}} d \mathbf{q}\left\langle\nabla_{\mathbf{q}} \Phi_{\mathbf{q}}|\times| \nabla_{\mathbf{q}} \Phi_{\mathbf{q}}\right\rangle
$$




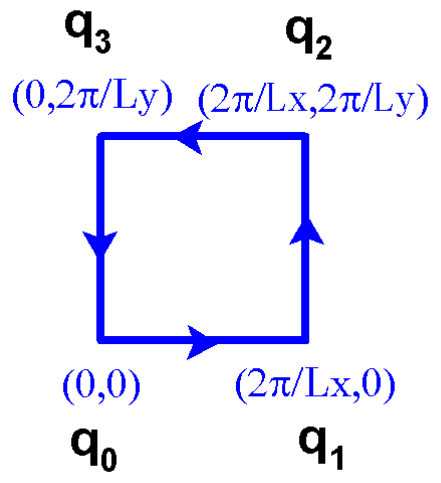

Figure 1. The rectangle $R_{\mathbf{q}}$ has a size $4 \pi^{2} / L_{x} L_{y}$, whose four vertices are denoted by $\mathbf{q}_{\alpha}(\alpha=0,1,2,3)$.

where the inner product includes a summation over $\left\{\mathbf{k}^{(0)}\right\}$, and $R_{\mathbf{q}}$ denotes the rectangle of $\left[0, \frac{2 \pi}{L_{x}}\right] \times\left[0, \frac{2 \pi}{L_{y}}\right]$.

Using the Stokes theorem, we rewrite Eq. (3) as a line integral

$$
C=\frac{1}{2 \pi i} \oint_{\partial R_{\mathbf{q}}} d \mathbf{l}_{q} \cdot\left\langle\Phi_{\mathbf{q}} \mid \nabla_{\mathbf{q}} \Phi_{\mathbf{q}}\right\rangle
$$

around the boundary of $R_{\mathbf{q}}$, denoted by $\partial R_{\mathbf{q}}$. The Chern number given in Eq. (4) is expressed as a winding number along closed path $\partial R_{\mathbf{q}}$. We can divide $\partial R_{\mathbf{q}}$ into $N$ small line segments with $\mathbf{q}_{\alpha}(\alpha=0,1 \cdots N)$ as their endpoints. In each segment, one can replace the derivatives in Eq. (4) by discrete differences and the integral by a summation, yielding

$$
C=\frac{1}{2 \pi} \sum_{\alpha=0}^{N-1} \operatorname{Arg}\left[\operatorname{det}\left(C_{\alpha, \alpha+1}\right)\right],
$$

where $\operatorname{Arg}(\cdot)$ stands for the principal argument, and $C_{\alpha, \alpha+1}$ is a $M \times M$ coupling matrix, 22] with elements $C_{\alpha, \alpha+1}^{m n}=\left\langle F_{\mathbf{q}_{\alpha}}^{m} \mid F_{\mathbf{q}_{\alpha+1}}^{n}\right\rangle$. Here, we have used the relation $\left\langle\Phi_{\mathbf{q}_{\alpha}} \mid \Phi_{\mathbf{q}_{\alpha+1}}\right\rangle=\operatorname{det}\left(C_{\alpha, \alpha+1}\right)$. Equivalently, one can first multiply the coupling matrices $\tilde{C}=\prod_{\alpha=0}^{N-1} C_{\alpha, \alpha+1}$, and then diagonalize $\tilde{C}$. The Chern number is given by the sum of the phases of the eigenvalues of $\tilde{C}$ divided by $2 \pi$.

In practice, it is sufficient to take $N=4$ and $\mathbf{q}_{\alpha}$ with $\alpha=0,1,2$, and $3\left(\mathbf{q}_{4}=\mathbf{q}_{0}\right)$ to be the four vertices of $R_{\mathbf{q}}$ shown in Fig. 1, when $L_{x} \gg 1$ and $L_{y} \gg 1$. It is interesting to notice that for these $\mathbf{q}_{\alpha}, \mathbf{k}^{(0)}+\mathbf{q}_{\alpha}$ still belong to the set of $\left\{\mathbf{k}^{(0)}\right\}$, so all the quantities that are needed for the calculation of the Chern number can be obtained in the system with periodic boundary conditions. Moreover, using the inverse FT, the matrix elements of the coupling matrix $C_{\alpha, \alpha+1}$ can be expressed in real space as

$$
C_{\alpha, \alpha+1}^{m n}=\left\langle\varphi_{\theta=0}^{m}\left|e^{i\left(\mathbf{q}_{\alpha}-\mathbf{q}_{\alpha+1}\right) \cdot \mathbf{r}}\right| \varphi_{\theta=0}^{n}\right\rangle .
$$

Here, $\varphi_{\theta=0}^{m}(\mathbf{r})$ with $m=0,1,2 \cdots(M-1)$ are the single-particle wave functions of the occupied electron states for periodic boundary conditions. The product of the coupling 
matrices

$$
\widetilde{C}=C_{0,1} C_{1,2} C_{2,3} C_{3,0}
$$

is first diagonalized to find $M$ eigenvalues denoted as $\lambda_{m}$. Since $\widetilde{C}$ is not Hamiltian, its eigenvalues $\lambda_{m}$ are usually complex numbers. Then the Chern number is given by

$$
C=\frac{1}{2 \pi} \sum_{m=0}^{M-1} \operatorname{Arg}\left(\lambda_{m}\right),
$$

where the range of the principal argument function $\operatorname{Arg}(\cdot)$ is taken to be $(-\pi, \pi]$. We mention that $\widetilde{C}$ tends to be diagonal when $M$ is sufficiently large [22], so the sum of the phases of all the diagonal elements in $\widetilde{C}$ divided by $2 \pi$ yields a good approximation to the Chern number.

Prodan, Hughes, and Bernevig [21] proposed an efficient numerical approach to the Chern number, by extending the formula for pure systems straightforwardly to disordered systems. Their expression is also in real space, which however involves multiple commutators of the coordinate operators and projection operator, and is unlikely reducible to a single term. Our method has the advantage that the formula contains only a single term and is more physically transparent. In the next two sections, numerical calculations will be carried out to show the validity and efficiency of this formula for calculating the Chern number.

\section{The Haldane Model}

Let us consider the Haldane model [3] for the quantum anomalous Hall effect defined on a honeycomb lattice, including a random on-site disorder potential

$$
H=-\sum_{\langle i, j\rangle} \hat{c}_{i}^{\dagger} \hat{c}_{j}+i t \sum_{\langle\langle i, j\rangle\rangle} v_{i j} \hat{c}_{i}^{\dagger} \hat{c}_{j}+\sum_{i} \omega_{i} \hat{c}_{i}^{\dagger} \hat{c}_{i}
$$

Here, the first term describes the usual nearest-neighbor hopping with the hopping integral being taken to be unity, and the second term stands for the next-nearestneighbor hopping with a complex hopping integral and $v_{i j}=\left(\boldsymbol{d}_{k j} \times \boldsymbol{d}_{i k}\right)_{z} /\left|\left(\boldsymbol{d}_{k j} \times \boldsymbol{d}_{i k}\right)_{z}\right|$. $i$ and $j$ are two next nearest neighbor sites and $k$ their common nearest neighbor. The vector $\boldsymbol{d}_{i k}$ points from $k$ to $i$, and the distance between two nearest neighbor sites is taken to be unity. The third term represents a random on-site potential, with $\omega_{i}$ uniformly distributed between $[W / 2, W / 2]$. It is known [3] that in the absence of disorder, the energy spectrum of the Haldane model has a middle band gap between $-3 \sqrt{3} t$ and $3 \sqrt{3} t$. When the Fermi energy lies in the band gap, the system shows a quantized Hall conductivity $e^{2} / h$, without an applied magnetic field.

Numerical calculation of the Chern number is carried out for a disordered system with size $\mathrm{N}=48 \times 48$ and $t=0.2$, and the results are shown in Fig. 2. In the absence of disorder $(W=0)$, the Chern number is well quantized to 1 within the middle band gap, which is consistent with the known result [3]. With increasing disorder strength, the $C=1$ plateau narrows, manifesting the levitation and pair annihilation [23] of extended states for the valence and conduction bands, whose Chern numbers have opposite signs. 


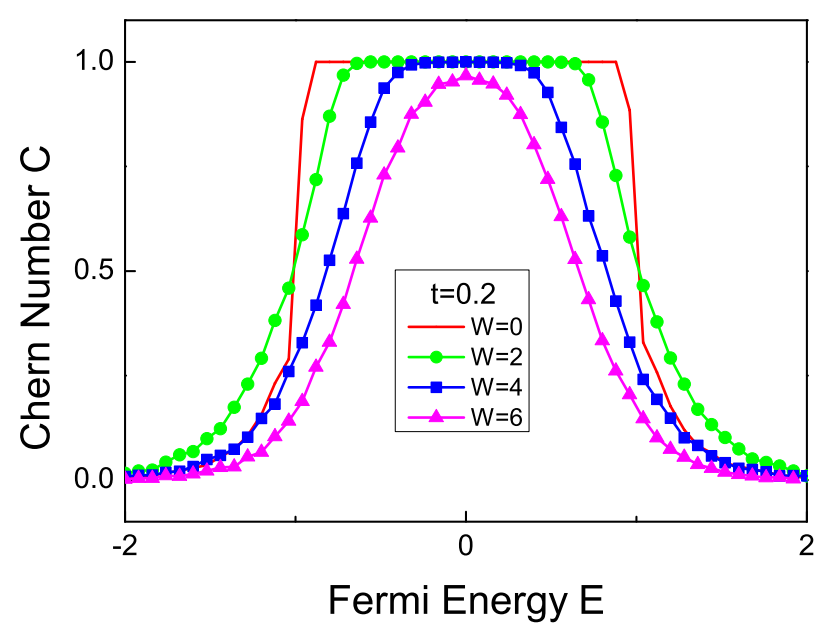

Figure 2. Chern number for the Haldane model as a function of electron Fermi energy $E$ for $t=0.2$, for several different disorder strengths. The result is averaged over 400 disorder configurations for sample size $N=48 \times 48$.

The calculated Chern number at a fixed Fermi energy $(E=0)$ for $t=0.1$ as a function of disorder strength is plotted in Fig. [3 for different sample sizes. It is found that the Chern number is robust against weak disorder $W<4$. With increasing $W$ from 4 to 6 , the Chern number continuously decreases to nearly zero. With increasing the sample size, the transition process becomes sharper and sharper, which conforms the expectation that the transition should become a sudden drop from 1 to 0 in the thermodynamic limit.

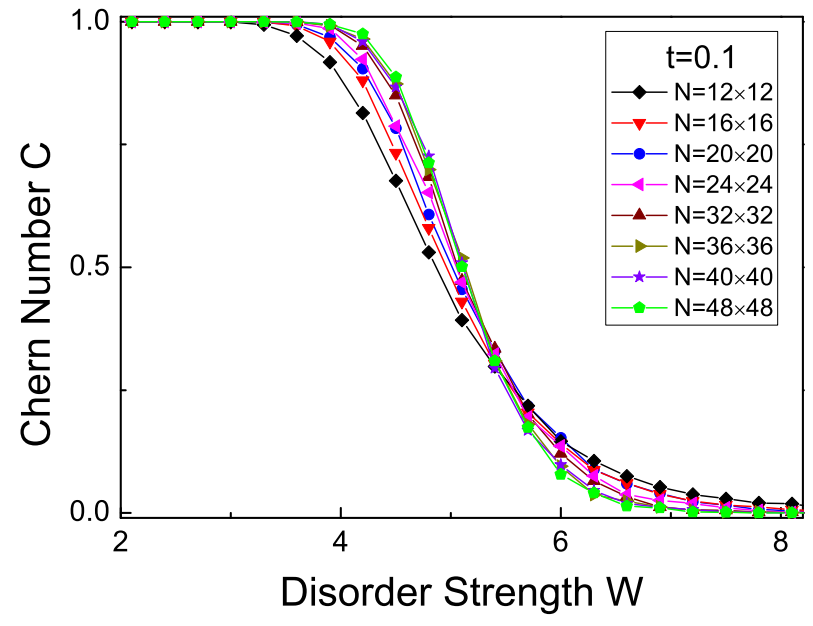

Figure 3. Chern number for the Haldane model as a function of disorder strength $W$ at $t=0.1$, for several different sample sizes. The result is averaged over 400 disorder configurations. 


\section{Hofstadter model}

We next turn to the Hofstadter model 24] on a 2D square lattice. Under an external uniform magnetic field with the Landau gauge $\vec{A}=(y B, 0,0)$, the model Hamiltonian is given by

$$
H_{0}=-\sum_{m, n}\left(e^{i n \phi} c_{m, n}^{\dagger} c_{m+1, n}+c_{m, n}^{\dagger} c_{m, n+1}\right)+H . c .,
$$

where integers $m$ and $n$ are the $x$ and $y$ coordinates of a lattice site, $c_{m, n}$ is the fermion annihilation operator on the site, and the hopping integral has been set to be the unit of energy. The magnetic flux quanta per plaquette is $\phi=2 \pi q / p$. In the absence of disorder, the original band splits into $p$ Landau levels for mutually prime integers $p$ and $q$. The special case of $q=1$ has been widely studied in previous works. [15, 16, 17, 25, 26, 27] It was found that each Landau band carries a Chern number +1 except the band at the center $(E=0)$ which carries a negative Chern number $-(p-1)$ for odd $p$ or $-(p / 2-1)$ for even $p$. Figure 4 shows the calculated Chern number as a function of the Fermi energy at $q / p=1 / 16$ for a sample of size $\mathrm{N}=64 \times 64$. We see that the Chern number of each Landau level is +1 , except the central two Landau levels, each one having a Chern number -7 . As a result, the sum of the Chern numbers of all the Landau levels in the full energy band is zero.

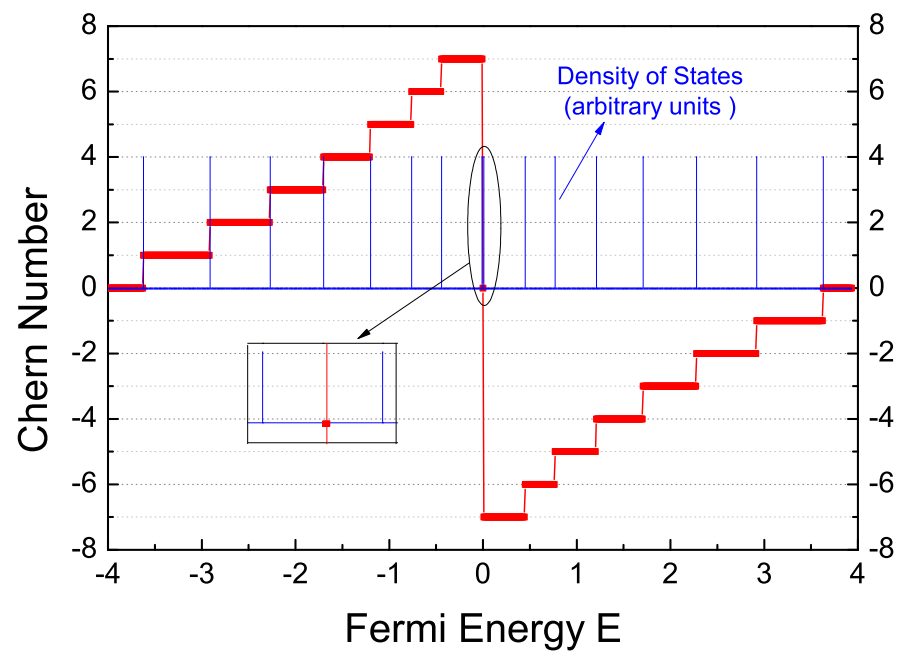

Figure 4. Calculated Chern number and electron density of states for the lattice Hofstadter model in the full energy band for magnetic flux $q / p=1 / 16$. The disorder strength is set to be $W=0$ and $N=64 \times 64$. The inset is a drawing of partial enlargement.

To study the disorder effect, we include into the Hamiltonian a random onsite potential of the form $\sum_{m, n} \omega_{m, n} c_{m, n}^{\dagger} c_{m, n}$, with $w_{m, n}$ randomly distributed between $[-W / 2, W / 2]$. The calculated Chern number at a fixed Fermi energy $E=-2.75$ is displayed as a function of disorder strength $W$ in Fig. 5. The Chern number is well quantized for weak disorder. With increasing $W$, the interesting direct transition [16] 


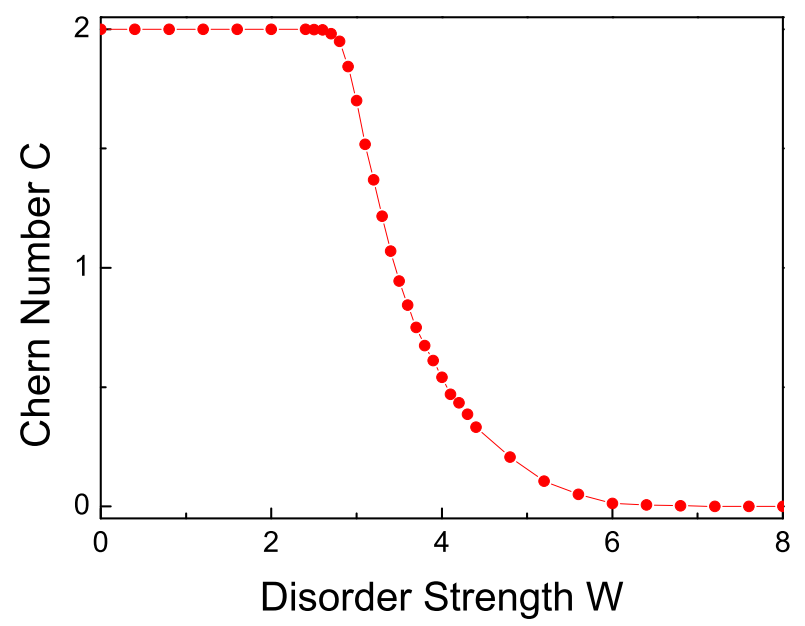

Figure 5. Chern number as a function of disorder strength $W$ for the lattice Hofstadter for magnetic flux $q / p=1 / 16$ and sample size $N=64 \times 64$. The result is averaged over 400 disorder configurations.

from the $C=2$ quantum Hall plateau to a trivial insulator state with $C=0$ is reproduced. Moreover, the critical disorder strength is estimated to be about $W=3.5$, in good agreement with the result obtained from the Kubo formula calculation [16].

\section{Summary}

To conclude, we have proposed an efficient coupling-matrix method for calculating the Chern number of disordered systems. The present approach is applied to both the Haldane model and lattice Hofstadter model. The calculated Chern number is found well quantized even if the sample size is not very large. The calculated results, in particular the disorder-induced phase transition, are in good agreement with the known results. Our approach can be directly applied to calculate the spin Chern numbers [11, 12] for the quantum spin Hall systems, from which the $\mathrm{Z}_{2}$ index can be extracted.

\section{ACKNOWLEDGMENTS}

This work is supported by the State Key Program for Basic Researches of China under Grants Nos. 2009CB929504 (LS), 2011CB922103, and 2010CB923400 (DYX), the National Natural Science Foundation of China under Grant Nos. 11225420, 11074110 (LS), 11074111 (RS), 11174125, 11074109,91021003 (DYX), Natural Science Foundation of Jiangsu Province in China under grant No. BK2010364 (YJ), and a project funded by the PAPD of Jiangsu Higher Education Institutions. We also thank the US NSF Grants No. DMR-0906816 and No. DMR-1205734, and Princeton MRSEC Grant No.DMR0819860 (DNS). 


\section{References}

[1] K. V. Klitzing, G. Dorda, and M. Pepper, Phys. Rev. Lett. 45, 494 (1980).

[2] D. C. Tsui, H. L. Stormer, and A. C. Gossard, Phys. Rev. Lett. 48, 1559 (1982).

[3] F. D. M. Haldane, Phys. Rev. Lett. 61, 2015 (1988).

[4] C. L. Kane and E. J. Mele, Phys. Rev. Lett. 95, 226801 (2005).

[5] B. A. Bernevig, T. L. Hughes, and S. C. Zhang, Science 314, 1757 (2006).

[6] M. Z. Hasan and C. L. Kane, Rev. Mod. Phys. 82, 3045 (2010).

[7] X. L. Qi and S. C. Zhang, Physics Today. 63, 33 (2010).

[8] D. J. Thouless, Topological Quantum Numbers in Nonrelativistic Physics (World Scientific, Singapore, 1998).

[9] D. J. Thouless, M. Kohmoto, M. P. Nightingale, and M. den. Nijs, Phys. Rev. Lett. 49, 405 (1982); D. J. Thouless, J. Phys. C 17, L325 (1984).

[10] Q. Niu, D. J. Thouless, and Y. S. Wu, Phys. Rev. B. 31, 3372 (1985).

[11] D. N. Sheng, Z. Y. Weng, L. Sheng, and F. D. M. Haldane, Phys. Rev. Lett. 97, 036808 (2006).

[12] E. Prodan, Phys. Rev. B 80, 125327 (2009); E. Prodan, New J. Phys. 12, 065003 (2010); Z. Xu, L. Sheng, D. Y. Xing, E. Prodan, D. N. Sheng, Phys. Rev. B 85, 075115 (2012).

[13] T. Fukui, Y. Hatsugai, and H. Suzuki, J. Phys. Soc. Jpn. 74, 1674 (2005).

[14] Y. Huo and R. N. Bhatt, Phys. Rev. Lett. 68, 1375 (1992).

[15] K. Yang and R. N. Bhatt, Phys. Rev. Lett. 76, 1316 (1996).

[16] D. N. Sheng and Z. Y. Weng Phys. Rev. Lett. 75, 2388 (1995); ibid 78, 318 (1997); ibid 80, 580 (1998).

[17] K. Yang and R. N. Bhatt, Phys. Rev. B 59, 8144 (1999).

[18] A. Kitaev, Ann. Phys. 321, 2 (2006).

[19] R. Bianco and R. Resta, Phys. Rev. B. 84, 241106(R) (2011).

[20] D. Ceresoli and R. Resta, Phys. Rev. B 76, 012405 (2007).

[21] E. Prodan, T. L. Hughes, and B. A. Bernevig, Phys. Rev. Lett. 105, 115501 (2010).

[22] M. Baer, Chem. Phys. Lett. 35, 112 (1975); M. Baer, Mol. Phys. 40, 1011 (1980); M. Baer, J. Phys. Chem. A 40, 3181 (2000).

[23] M. Onoda, Y. Avishai and N. Nagaosa, Phys. Rev. Lett. 98, 076802 (2007).

[24] D. R. Hofstadter, Phys. Rev. B. 14, 2239 (1976).

[25] D. Z. Liu, X. C. Xie, and Q. Niu, Phys. Rev. Lett. 76, 975 (1996).

[26] X. C. Xie, D. Z. Liu, B. Sundaram, and Q. Niu, Phys. Rev. B. 54, 4966 (1996).

[27] T. Ando, Phys. Rev. B. 40, 5325 (1989). 